\title{
Hepatocellular carcinoma with pancreatic mass as the first symptom: a case report and literature review
}

\author{
Yue Zhang ${ }^{1,2 \#}$, Tao Han ${ }^{1 \#}$, Di Wang ${ }^{3 \#}$, Gao Li ${ }^{1,4 \#}$, Yanming Zhang ${ }^{1,2}$, Xiaodan Yang ${ }^{1}$, Tingsong Chen ${ }^{5}$, \\ Zhendong Zheng ${ }^{1}$
}

${ }^{1}$ Department of Oncology, Cancer Center, General Hospital of Northern Theater Command, Shenyang 110016, China; ${ }^{2}$ Postgraduate College, Jinzhou Medical University, Jinzhou 121001, China; ${ }^{3}$ Department of Pathology, General Hospital of Northern Theater Command, Shenyang 110016, China; ${ }^{4}$ Department of Clinical Pharmacy, Shenyang Pharmaceutical University, Shenyang 110016, China; ${ }^{5}$ Department of Invasive Technology, The Seventh People's Hospital of Shanghai University of Traditional Chinese Medicine, Shanghai 200137, China

\#These authors contributed equally to this work.

Correspondence to: Dr. Zhendong Zheng. Department of Oncology, Cancer Center, General Hospital of Northern Theater Command, No. 83 Wenhua Road, Shenyang 110016, China. Email: zhengzhdong@163.com; Dr. Tingsong Chen. Department of Invasive Technology, The Seventh People's Hospital of Shanghai University of Traditional Chinese medicine, Shanghai 200120, China. Email: cts552052597@163.com.

\begin{abstract}
Hepatocellular carcinoma (HCC) and primary pancreatic cancer are common malignant tumors of the digestive system. However, there are significant differences in treatment methods, medication types, and survival prognoses. For patients whose imaging findings suggest there to be a significant pancreatic mass and multiple masses in the liver, it can be easily misdiagnosed as a primary pancreatic cancer with liver metastasis in the clinic instead. Therefore, patients with a high likelihood of primary pancreatic cancer based on their clinical data, the pathological diagnosis should be confirmed through a needle biopsy as early as possible to avoid a misdiagnosis and possible mistreatment. In this study, our department admitted a patient with HCC that was characterized by a pancreatic occupation. The clinical data originally highly suggested primary pancreatic cancer with liver metastasis, but the final pathological puncture results had confirmed pancreatic metastasis of HCC. This case reminds us that a pathological biopsy should still be used as the final means of definite diagnosis and should be widely popularized for pancreatic nodules.
\end{abstract}

Keywords: Hepatocellular carcinoma (HCC); pancreatic cancer; pancreas metastasis; diagnosis; case report

Submitted Oct 18, 2019. Accepted for publication Nov 05, 2019.

doi: 10.21037/apm.2019.11.14

View this article at: http://dx.doi.org/10.21037/apm.2019.11.14

\section{Introduction}

Hepatocellular carcinoma (HCC), or liver cancer, is one of the most common malignant tumors. An extrahepatic metastasis most commonly accompanies hematogenous metastasis, and it can be transferred to the lungs, bones, distant lymph nodes, and brain (1). According to the current data, a pancreatic metastasis of HCC is quite rare. Correspondingly, pancreatic cancer, another malignant tumor of the digestive system, is prone to metastasize in the early stage, most commonly in the liver (2). Our department treated one case of HCC characterized by pancreatic occupation. Before the pathological diagnosis was determined, all imaging examinations and tumor marker changes had met the clinical diagnostic criteria of primary pancreatic cancer. However, the results of the biopsy showed a rare pancreatic metastasis of HCC. The patient's diagnosis process is reviewed below.

\section{Case presentation}

The patient in the case was a 70 -year-old man who was admitted to our department due to abdominal distension for 1 month in October 2017. He had mild abdominal distension since the beginning of 2017 without an apparent cause, but there was no visible edema in either eyelid 
or the lower extremities at that time, which led to the patient not giving much notice to the condition. The abdominal distension worsened in September, and urine volume decreased with lower extremity edema, anorexia, but no nausea and vomiting. The magnetic resonance cholangiopancreatography (MRCP) performed by the local hospital showed abnormal shape and signal of the pancreas, which was further confirmed and treated in our department. The patient had a history of being a hepatitis B virus carrier and recently was found to be in the active phase of the hepatitis $B$ virus.

During the physical examination, it was noted that there was no yellow staining of the skin, no yellow staining of the sclera, no liver palms, no spider mites, and no subcutaneous nodules. The superficial lymph nodes of the whole body were not swollen to the touch. The abdomen was mildly bulging and soft, with no tenderness and rebound pain. A mass of about $4 \times 5 \mathrm{~cm}$ in size could be felt in the upper abdomen. The liver and spleen could not be felt under the ribs, and the gallbladder could also not be felt by touch. Murphy's sign was (-), and there was tenderness in the liver and gallbladder areas (-), with mild edema in both lower extremities.

For the laboratory examination, the results of the test indicated that the tumor marker, carcinoembryonic antigen (CEA), was normal, alpha-fetoprotein (AFP) was $16.50 \mathrm{IU} / \mathrm{mL}$ (reference $0.0-7.0 \mathrm{ng} / \mathrm{mL}$ ), and carbohydrate antigen 19-9 (CA19-9) was 250.1 U/mL (reference 0.0-30.0 U/mL). For the coagulation parameters, the D dimer was $1.87 \mathrm{mg} / \mathrm{L}$, plasma fibrin degradation products were $6.70 \mathrm{mg} / \mathrm{L}$, activated partial thromboplastin time (APTT) was 40.2 seconds, prothrombin time (PT) was 16.7 seconds, and antithrombin III was $69 \%$. For the blood cell analysis, the platelet (PLT) count was $80 \times 10^{9} / \mathrm{L}$, and red blood cell (RBC) count was $4.01 \times 10^{9} / \mathrm{L}$. For the liver function test, serum total bile acid determination was $20.8 \mu \mathrm{mol} / \mathrm{L}$, serum direct bilirubin determination was $19.6 \mu \mathrm{mol} / \mathrm{L}$, serum total bilirubin determination was $31.6 \mu \mathrm{mol} / \mathrm{L}$, serum albumin determination was $25.9 \mathrm{~g} / \mathrm{L}$, and serum R-glutamyl transpeptidase was $177.85 \mathrm{U} / \mathrm{L}$; serum aspartate aminotransferase was $59.80 \mathrm{U} / \mathrm{L}$, and serum alkaline phosphatase was $287.35 \mathrm{U} / \mathrm{L}$. N-terminal B-type natriuretic peptide was $632.7 \mathrm{pg} / \mathrm{mL}$. Hepatitis B surface antigen, e antigen, and c antibody were positive.

The image examination results are described below. Abdominal ultrasound tips were as follows: (I) pancreatic head cancer; (II) nodular cirrhosis; (III) secondary gallbladder changes, gallbladder enlargement, and gallbladder deposits. PET/CT (Figure 1A,B) results suggested the following: (I) a full pancreatic head, uneven density, and uneven metabolism, considering the possibility of malignant lesions (pancreatic cancer); (II) liver cirrhosis, splenomegaly; (III) portal hypertension; (IV) ascites. The results of magnetic resonance enhancement indicated nodules of metastatic carcinoma, which suggested liver metastasis of primary pancreatic cancer (Figure 1C). The preliminary clinical diagnosis was as follows: (I) pancreatic cancer (malignant possibility), (i) liver metastasis; (II) nodular cirrhosis; (III) splenomegaly; (IV) ascites.

According to the results of PET/CT and abdominal ultrasound, the patient was highly suspected of having primary pancreatic cancer clinically, but we decided to make a further treatment plan after pathological a diagnosis for the sake of caution. The patient was asked to fast and abstain from drinking liquids before surgery, given ulinastatin and somatostatin to inhibit secretion of pancreatic juice, and was also provided intravenous nutritional support. On December 13, 2017, we performed a multipoint pancreatic biopsy on the patient. The pathological results showed that several mildly shaped small round cells were seen in the punctured tissue. Tumor thrombus was also seen in the vein. Immunohistochemistry results (Figure 2 ) showed the following: CK8/18(+), Hep (+), AFP (+), CK19(-), Syn (-), CD34(+), CD56(-), CgA (-), CEA (-), CA19-9(-), Ki67(+) about $10 \%$ CK (-), and CK20 (partial $+/-)$. According to the immunohistochemistry and morphology, pathological diagnosis suggested pancreatic metastasis of HCC.

The pathological results, combined with those of immunohistochemistry, confirmed that the patient had pancreatic metastasis of HCC. According to the results of the pancreatic biopsy, the patient had a precise diagnosis: (I) HCC; (i) pancreatic metastasis; (II) nodular cirrhosis; (III) splenomegaly; (IV) ascites. After the diagnosis was precise, we applied for a consultation with a surgical specialist. Considering the patient's poor liver function, the specialist believed that the risk of failure of the surgery was extremely high and did not recommend surgical treatment. We recommended the patient to take Sorafenib $400 \mathrm{mg}$ twice a day for first-line targeted therapy, and he was then discharged from the hospital. After follow-up, we learned that the patient's overall survival time was 11 months.

\section{Discussion}

HCC is a primary malignant tumor derived from hepatocytes, accounting for about $90 \%$ of all primary liver cancers (3). 

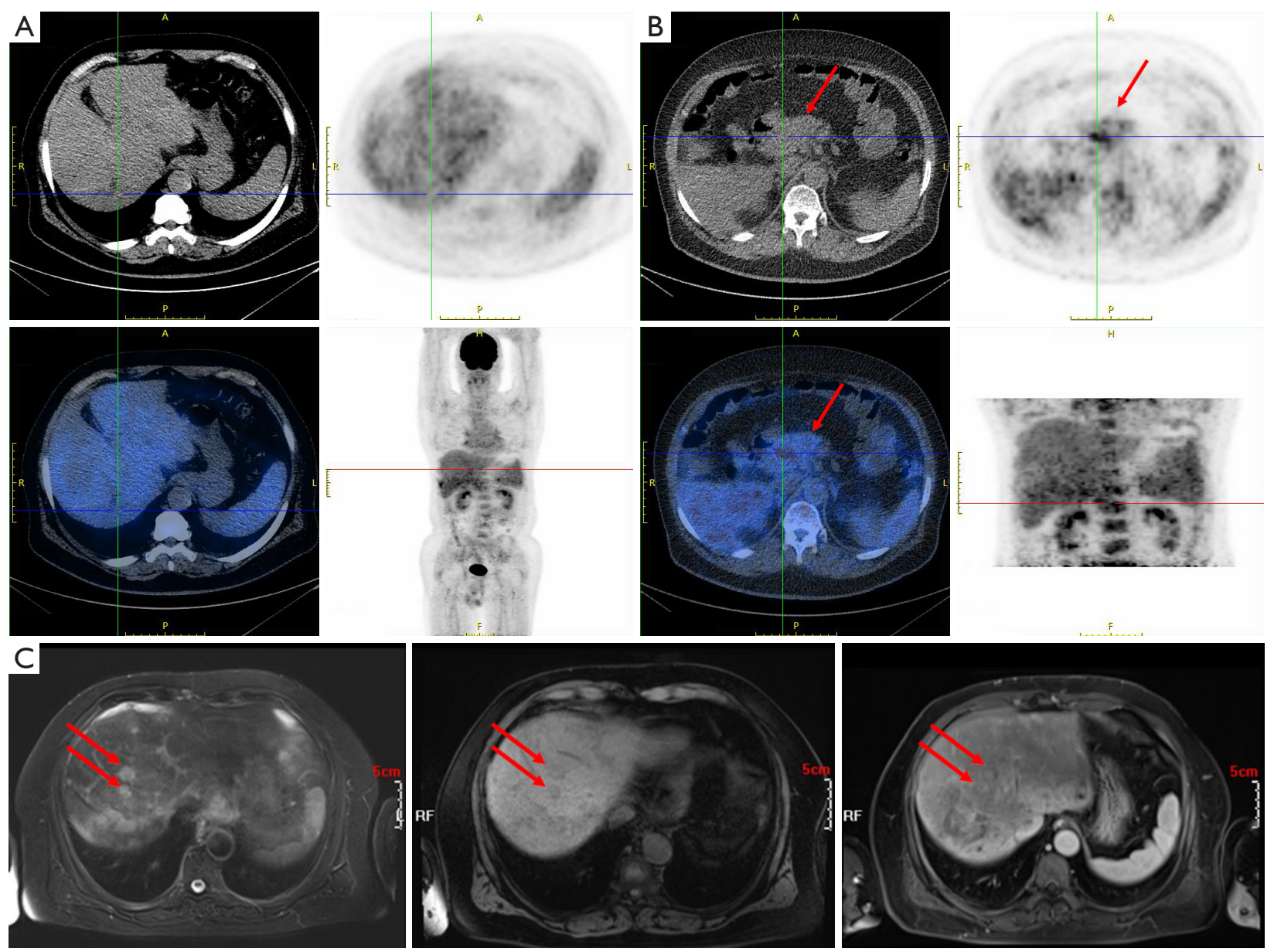

Figure 1 The liver's volume was reduced, the edge of the liver was uneven, and there was no obvious abnormality in radioactivity uptake (A). The pancreatic head is thickening about $6.1 \mathrm{~cm} \times 4.3 \mathrm{~cm}$ size with the density uneven, and the radioactivity uptake increased (B: red arrow): SUVmax: 3.8, delayed imaging SUVmax: 4.8. liver metastasis (C).

Globally, HCC is the fourth leading cause of cancer-related death, and its incidence is increasing annually (4). Hepatitis B, hepatitis $\mathrm{C}$, and cirrhosis from various causes are still some of the most common risk factors for HCC (5). As China has a high incidence of $\mathrm{HBV}$, it accounts for more than $50 \%$ of the world's liver cancer incidence $(4,5)$. HCC lacks typical clinical symptoms and signs in the early stage. Symptoms can come from liver cancer or hepatitis and cirrhosis background. It is challenging to distinguish HCC from benign liver disease. The detection rate of early liver cancer is not high in correlative imaging examination, and some patients have had extrahepatic metastasis when diagnosed preliminarily, which is already in the middle and late stage of the disease, and the prognosis is poor (6). Extrahepatic metastasis of primary liver cancer is most common in hematogenous metastasis, mostly to the lungs, bones, distant lymph nodes, and brain. ${ }^{1}$ The incidence of extrahepatic lung metastasis in HCC is nearly $30 \%$ (7), but the occurrence of pancreatic metastasis in HCC is very low, with only a few reported in case reports (8-11), the first of which was by Lowe et al. (8) in 1997. Research by Cheng (12) shows that the overall survival of patients with advanced liver cancer who were not given intervention was only 7.9 months, while the overall survival of patients taking sorafenib could be extended to 10.7 months.

Pancreatic cancer is another digestive system tumor with high morbidity and high mortality. Sixty percent of patients have distant metastasis at the time of the first diagnosis, of which the liver is the most common, and $25 \%$ of patients are locally advanced. Radical resection is not possible in these patients, exhibiting a median survival of 

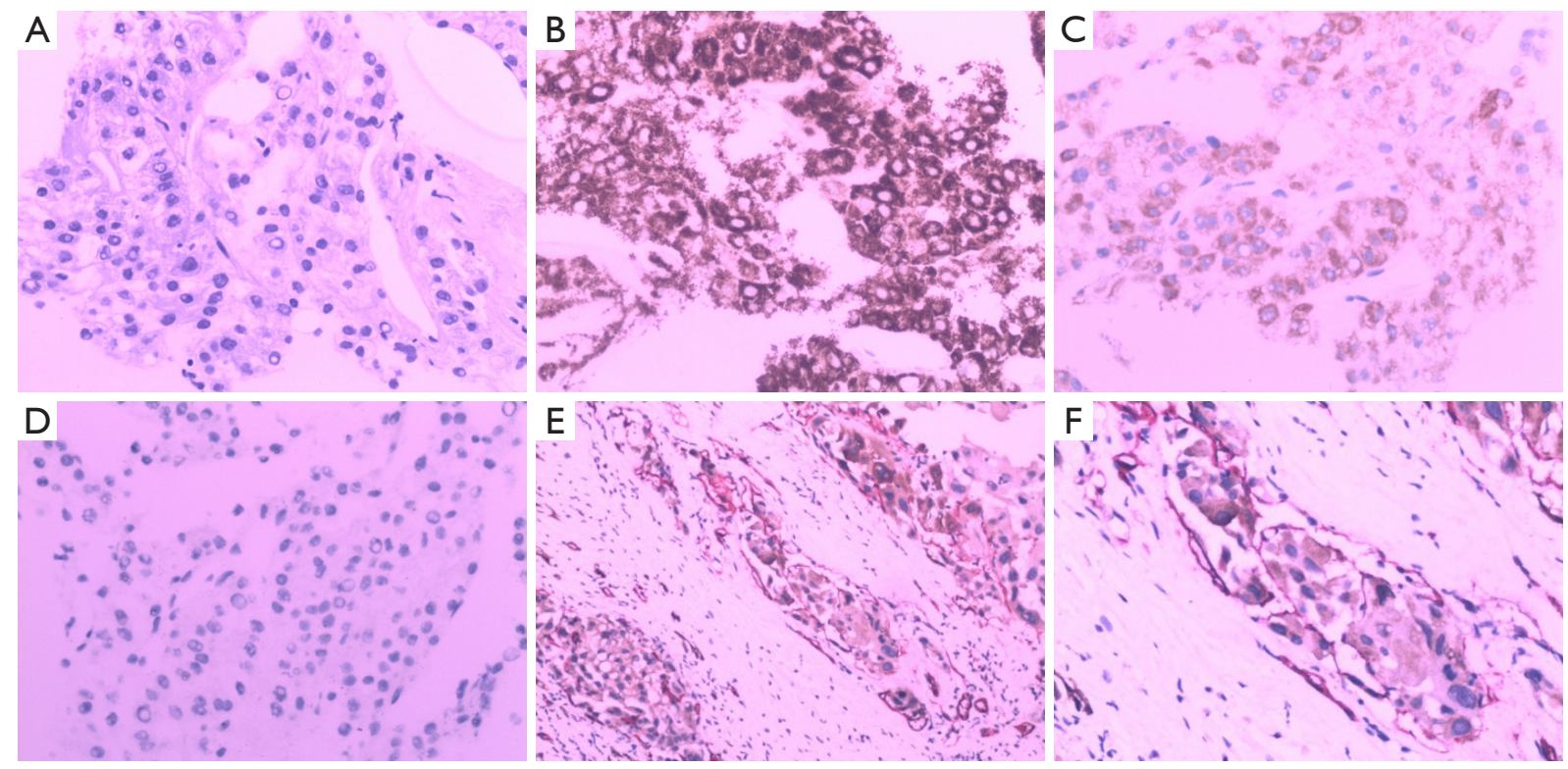

Figure 2 Hematoxylin and eosin stains showed poorly differentiated malignant cells in the pancreatic masses (A); immunohistochemical stains showed positive staining of tumor cells for Hep (B), AFP (C) and negative staining of that for CA19-9 (D). Tumor thrombus was also seen in the vein $(\mathrm{E}, \mathrm{F})(\times 200)$.

only 6 to 9 months $(13,14)$. Compared with simple lung metastasis or lymph node metastasis, patients combined with liver metastasis often have a worse prognosis, and their 5 -year survival rate is only about $6 \%$ (2), so early diagnoses and treatment are particularly important. On the other hand, patients with primary pancreatic cancer lack typical clinical symptoms and auxiliary examinations. The clinical and imaging findings of most patients with metastatic pancreatic cancer are like those of primary pancreatic cancer, but there are significant differences in the prognosis and treatment choice between them. Therefore, the differential diagnosis of metastatic pancreatic carcinoma and primary pancreatic cancer is particularly important.

In terms of treatment, advanced liver cancer is often treated with systemic therapy, especially with molecularly targeted therapy led by sorafenib. For metastatic pancreatic cancer, except that simultaneous resection of primary tumor and metastasis is still attempting for a few pancreatic cancers with solitary liver metastasis $(15,16)$, FOLFIRONOX-based systemic chemotherapy has replaced gemcitabine and is considered to be the first-line standard treatment for patients with metastatic pancreatic cancer (17). In this report, we note three points that led us to make the misdiagnosis of advanced pancreatic cancer. At first, the patient's ultrasound, PET/CT, and enhanced magnetic resonance did not show a clear malignant mass in the liver. Secondly, the increase of tumor marker AFP was not significant, while CA19-was is significantly increased. Lastly, both PET/CT and abdominal ultrasound showed a high possibility of pancreatic cancer with liver metastasis. Therefore, based on clinical statistics, imaging findings, and serum tumor marker changes, HCC with pancreatic metastasis for this patient was easily misdiagnosed as primary pancreatic cancer. Finally, for this patient, we chose to perform a needle biopsy on the mass in the head of the pancreas for cautions' sake. The pathological results confirmed that the tumor originated from HCC. This also reminds us that pathological diagnosis plays an essential role in the diagnosis of pancreatic tumors and is related to the patient's therapeutic response and survival.

In addition, CA19-9, as a used serological marker, is one of the essential tumor markers in the diagnosis of pancreatic cancer. However, studies show that CA19-9 may also increase in biliary tract infections, biliary obstruction, and other digestive system malignancies (18), which means that CA19-9 is not sufficiently specific in the diagnosis of pancreatic cancer. We suggest that imaging results such as CT, ultrasound, or even PET/CT be considered crucial references in the diagnosis of pancreatic tumors, but the final molecular pathology should still be the gold standard for diagnosis. 


\section{Conclusions}

In a review of the diagnosis process for patients, we have summarized the following experiences; although the AFP of the patients increased slightly and there was no primary apparent malignant mass in the liver, the history showed that the patient had been carrying the hepatitis B virus for more than 20 years. The most recent test results indicated that the hepatitis $B$ virus was in an active stage with the hepatitis B surface antigen, e antigen, and c antibody all positive. Hepatitis $\mathrm{B}$ virus infection is a common cause of HCC, and China is a country with a high incidence rate of the hepatitis B virus infection. The patient has had cirrhosis for several years at the time of medical treatment. At the same time, there is the possibility of having small liver cancer in the context of hepatitis cirrhosis. Liver cirrhosis nodules and small liver cancer are difficult to identify by either ultrasound or enhanced MR, which is one of the most likely causes of malignant nodules in the liver in our case report. The patient's immunohistochemistry suggests CD34+, and the pathological morphology suggests a tumor thrombus in the vasculature, suggesting the possibility of small liver cancer transferring to the pancreas through the blood. Therefore, for patients who have a hepatitis $B$ virus infection, pancreatic mass but no focal primary liver malignant lesions, and whose tumor markers also change indistinctively, the possibility of HCC should be considered before the biopsy pathology is confirmed even if imaging results strongly indicate primary pancreatic cancer. Pathological biopsy should still be used as the final means of definite diagnosis and should be widely popularized for differentiating primary $\mathrm{HCC}$ with pancreatic metastasis and primary pancreatic cancer. Moreover, the overall survival of the patient in this report was 11 months, which was longer than that of the patients in the sorafenib group studied by Cheng et al. This proves the accuracy of the diagnosis and the effectiveness of the treatment.

This article reports a rare case of HCC with pancreatic metastasis. The case was characterized by a pancreatic mass, slight AFP elevation, and unclear imaging of the liver lesions, all of which made a misdiagnosis of pancreatic cancer highly likely. Fortunately, we confirmed the pathology by a puncture biopsy and gave the patient a correct treatment recommendation, which fully confirms the vital role of molecular pathology in the diagnosis of malignancies.

\section{Acknowledgments}

Funding: This work was supported by the National Natural Science Foundation of China under Grant number 81702622).

\section{Footnote}

Conflicts of Interest: The authors have no conflicts of interest to declare.

Ethical Statement: The author is accountable for all aspects of the work in ensuring that questions related to the accuracy or integrity of any part of the work are appropriately investigated and resolved. All clinical investigations were approved by the General Hospital of Northern Theater Command, and written informed consent was obtained from the patient.

\section{References}

1. Natsuizaka M, Omura T, Akaike T, et al. Clinical features of hepatocellular carcinoma with extrahepatic metastases. J Gastroenterol Hepatol 2005;20:1781-7.

2. Shi S, Yu XJ. Time to think: Selecting patients who may benefit from synchronous resection of primary pancreatic cancer and liver metastases. World J Gastroenterol 2018;24:3677-80.

3. European Association for the Study of the Liver. EASL Clinical Practice Guidelines: Management of hepatocellular carcinoma. J Hepatol 2018;69:182-236.

4. Global Burden of Disease Liver Cancer Collaboration, Akinyemiju T, Abera S, et al. The Burden of Primary Liver Cancer and Underlying Etiologies From 1990 to 2015 at the Global, Regional, and National Level: Results From the Global Burden of Disease Study 2015. JAMA Oncol 2017;3:1683-91.

5. Chen W, Zheng R, Baade PD, et al. Cancer statistics in China, 2015. CA Cancer J Clin 2016;66:115-32.

6. Lee JI, Kim JK, Kim DY, et al. Prognosis of hepatocellular carcinoma patients with extrahepatic metastasis and the controllability of intrahepatic lesions. Clin Exp Metastasis 2014;31:475-82.

7. $\mathrm{Wu} W, \mathrm{He} X$, Andayani D, et al. Pattern of distant extrahepatic metastases in primary liver cancer: a SEER 
based study. J Cancer 2017;8:2312-8.

8. Lowe CJ Jr, Riepe SP, Wood WC. Hepatocellular carcinoma presenting as a pancreatic head mass: report of an unusual case. Am J Clin Oncol 1997;20:509-10.

9. Texler ML, Pierides J, Maddern GJ. Case report: A hepatocellular carcinoma metastasis in the distal pancreas. J Gastroenterol Hepatol 1998;13:467-70.

10. Sugai Y, Watanabe Y, Hasoya T, et al. Pancreatic metastasis from hepatocellular carcinoma. AJR Am J Roentgenol 1999;172:839-40.

11. Xiong J, Kwong Chian S, Li J, et al. Iodine-125 seed implantation for synchronous pancreatic metastases from hepatocellular carcinoma: A case report and literature review. Medicine (Baltimore) 2017;96:e8726.

12. Cheng AL, Kang YK, Chen Z, et al. Efficacy and safety of sorafenib in patients in the Asia-Pacific region with advanced hepatocellular carcinoma: a phase III randomised, double-blind, placebo-controlled trial. Lancet
Oncol 2009;10:25-34.

13. Jemal A, Bray F, Center MM, et al. Global cancer statistics. CA Cancer J Clin 2011;61:69-90.

14. Witkowski ER, Smith JK, Tseng JF. Outcomes following resection of pancreatic cancer. J Surg Oncol 2013;107:97-103.

15. Zanini N, Lombardi R, Masetti M, et al. Surgery for isolated liver metastases from pancreatic cancer. Updates Surg 2015;67:19-25.

16. Hackert T, Niesen $W$, Hinz $U$, et al. Radical surgery of oligometastatic pancreatic cancer. Eur J Surg Oncol 2017;43:358-63.

17. Conroy T, Desseigne F, Ychou M, et al. FOLFIRINOX versus gemcitabine for metastatic pancreatic cancer. $\mathrm{N}$ Engl J Med 2011;364:1817-25.

18. Marrelli D, Caruso S, Pedrazzani C, et al. CA19-9 serum levels in obstructive jaundice: clinical value in benign and malignant conditions. Am J Surg 2009;198:333-9.
Cite this article as: Zhang Y, Han T, Wang D, Li G, Zhang Y, Yang X, Chen T, Zheng Z. Hepatocellular carcinoma with pancreatic mass as the first symptom: a case report and literature review. Ann Palliat Med 2019;8(5):740-745. doi: 10.21037/apm.2019.11.14 